\title{
Mermelada hipocalórica de arazá y babaco
}

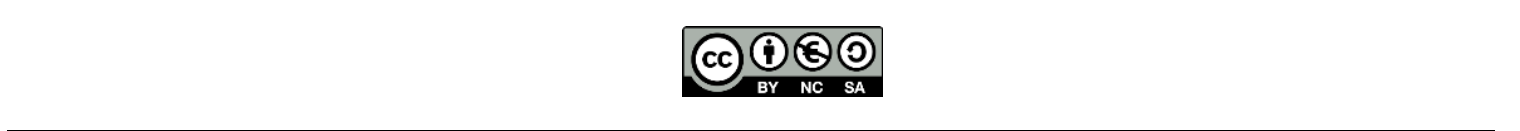

Hypocholoric jam from arazá and babaco

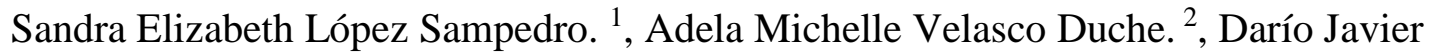

Baño Ayala. ${ }^{3} \&$ Armando Vinicio Paredes Peralta. ${ }^{4}$

\begin{abstract}
.
The study was carried out at the ESPOCH Faculty of Livestock Sciences, where prepare a jam using arazá and babaco in order to provide added value to these fruits, using stevia at different levels 2, 4, 6\%, as a substitute for sugar, for the statistical analysis a completely randomized design (DCA) of four treatments with four repetitions and $200 \mathrm{~g}$ per experimental unit was applied, the Acidity, reducing sugars and Brix presented statistically significant differences with a significance of $\mathrm{p}<0.05$. Level $6 \%$ shows better nutritional characteristics. Protein 1.33\%, Fiber 3.29\%, Ethereal Extract 0.19\%, Ash $0.28 \%$, Non-Nitrogen Free Extract 5.19\% and lower caloric value $17.81 \mathrm{Kcal} / \mathrm{g}$. No significant differences were found for Vitamin $\mathrm{C}$ or microorganisms were reported, the product with the highest sensory acceptance was the $6 \%$ level and with a cost benefit of 1.30 in US dollars. It is recommended to use level $6 \%$ stevia in the production.
\end{abstract}

DOI: https://doi.org/10.33262/concienciadigital.v3i3.1287

Keywords: Jam, arazá, stevia, nutrition.

\section{Resumen.}

El estudio se llevó a cabo en la Facultad de Ciencias Pecuarias ESPOCH, donde se elaboró una mermelada de arazá y babaco; con el objetivo de aportar valor agregado a

\footnotetext{
${ }^{1}$ Escuela Superior Politécnica de Chimborazo, Facultad de Ciencias Pecuarias. Riobamba, Ecuador.salopez@espoch.edu.ec

${ }^{2}$ Profesional Independiente, Riobamba, Ecuador. wichyad@gmail.com

${ }^{3}$ Escuela Superior Politécnica de Chimborazo, Facultad de Ciencias Pecuarias. Riobamba, Ecuador. dbano@yahoo.es

${ }^{4}$ Escuela Superior Politécnica de Chimborazo, Facultad de Ciencias Pecuarias. Riobamba, Ecuador. armando.paredes@espoch.edu.ec
} 
estas frutas, utilizando stevia en diferentes niveles 2, 4, $6 \%$, como sustituto del azúcar blanco, para el análisis estadístico se aplicó un diseño complemente al azar (DCA) de cuatro tratamientos con cuatro repeticiones y $200 \mathrm{~g}$ por unidad experimental, la acidez, azúcares reductores y Brix presentaron diferencias estadísticamente significativas para una significancia $\mathrm{p}<0,05$. El nivel $6 \%$ muestra mejores características nutricionales Proteína 1,33 \%, Fibra 3,29 \%, Extracto Etéreo 0,19\%, Cenizas 0,28 \%, Extracto libre no Nitrogenado 5,19\% y menor valor calóricos 17,81 Kcal/ g. No se encontraron diferencias significativas para Vitamina $\mathrm{C}$ ni se reportaron microorganismos, el producto de mayor aceptación sensorial fue el nivel $6 \%$ y con un beneficio costo de 1,30 en dólares americanos. Se recomienda utilizar el nivel 6\% stevia en la elaboración de mermeladas y otros productos dietéticos para personas con diabetes y problemas nutricionales.

Palabras clave: mermelada, arazá, stevia, nutrición.

\section{Introducción.}

El Basándose en el concepto de mermelada según la (NTE INEN 2825. 2013, la cual manifiesta que "es el producto obtenido por la concentración de la pulpa, con cantidades adecuadas de azúcar, pectina y acido, debe caracterizarse por una buena consistencia, presentar un cuerpo pastoso, pero no duro y tener su dulzor adecuado" se formuló un producto con las características citadas en la Norma. (Rojas 2007, citada en Velasco A 2020)

El arazá es una fruta aromática poco utilizada pero con muy buenas características físico químicas y un elevado potencial industrial (Duran S, 2012) por otra parte el babaco otro fruto poco industrializado ayudará a reducir los niveles de acidez que posee el arazá, contiene abundante agua (alrededor del 93\%), su porcentaje de azucares es bajo al igual que su aporte calórico, tiene un buen perfil vitamínico y un sabor agradable, (Espín E, 2016) además de poseer una enzima llamada papaína la cual es digestiva y facilita el desdoblamiento de la proteína animal.(Cuellar E, 2013, citada en Velasco A 2020)

La Stevia presenta un sabor particular al comienzo y una duración más prolongada, aunque algunos de sus extractos pueden tener un sabor amargo similar al "regaliz" en altas concentraciones, debido a que esta es 300 veces más dulce que el azúcar o sacarosa, trabajando en $\mathrm{pH}$ de 3 a 9 que son estables y con temperaturas de $100^{\circ} \mathrm{C}$ en el procesamiento de alimentos, si se sobrepasan estas temperaturas se pierde su dulzor. (Rojas S, 2007).

Se pretende entonces elaborar una mermelada hipocalórica de arazá y babaco utilizando diferentes niveles de stevia (stevia rebaudiana) en la formulación 2, 4 y $6 \%$ evaluándola de nutricional, sensorial, microbiológica y económicamente. 


\section{Metodología.}

El producto se elaboró y se analizó en la Facultad de Ciencias Pecuarias de la Escuela Superior Politécnica de Chimborazo, ubicada en la Panamericana Sur Km 1 1/2 con una duración de 120 días.

Las mediciones que se realizaron fueron $\mathrm{pH}$, Acidez, ${ }^{\circ}$ Brix y Azúcares Reductores, Humedad, Proteína, Fibra, Extracto Etéreo, Minerales, Carbohidratos, Valor Calórico, Vitamina C, Mohos y Levaduras (UFC/g), color, sabor, olor

\section{Elaboración de la mermelada}

Se recolectó las frutas (arazá y babaco) en su estado óptimo de maduración verde ligeramente amarillento, para proceder a la selección eliminando las frutas en mal estado. Luego se desinfectaron y se eliminaron las impurezas o material extraño. Se pesó la materia prima y los ingredientes para la formulación.

Se procedió a la cocción de las frutas agregando la pectina y el ácido cítrico para ajustar el pH, en el caso del tratamiento testigo se esperó a que los ${ }^{\circ}$ Brix llegaran de 65 a 68, en la mermelada baja en calorías los ${ }^{\circ}$ Brix hasta 40 , se procedió luego a su envasado mientras la mermelada esta aproximadamente a $85^{\circ} \mathrm{C}$.

Se enfrió la mermelada por inmersión, aspersión o rociada su almacenamiento se realiza en lugar fresco y seco (bajo sombra) evitando la luz directa (Espín, 2012. citada en Velasco A 2020).

\section{Determinación de $\mathrm{pH}$.}

Se realizó mediante el uso de un pH- metro digital previamente calibrado con solución buffer a 7 y se utilizó el método descrito en la AOAC 981.12.

\section{Determinación de Acidez}

Se realizó por medio de volumetría acido-base, mediante el método (AOAC 942.15 A).

\section{Determinación de Azúcares reductores.}

Se realizó por el método de Fehling.

\section{Determinación de Solidos solubles}

Se mide mediante un refractómetro a $20^{\circ} \mathrm{C}$, utilizando el método (AOAC 920.151)

\section{Determinación de Humedad}

Se determinó mediante el método (AOAC, 10.184). 


\section{Determinación Proteína}

Contenido de proteína, para la determinación de proteína donde se utilizó el método de Kjeldahl, bajo la norma (AOAC 920.152).

\section{Determinación de Fibra}

Para la fibra se determinó mediante el método que consiste en la digestión acida y alcalina bajo la norma (AOAC 985.29).

Determinación de Extracto Etéreo

Para el extracto etéreo se determinó mediante el método Soxhlet, bajo la norma (AOAC 31.4.02, 2000).

\section{Determinación de Minerales}

Para cenizas se realizó mediante la incineración por mufla, bajo la norma (AOAC 940.26).

\section{Determinación de Carbohidratos o ELnN}

Para Extracto Libre Nitrogenado se obtuvo por diferencian entre 100 y la suma de los otros componentes proteína, humedad, cenizas, extracto etéreo, fibra cruda.

Determinación del contenido Calórico (Kcal/g)

Para el contenido calórico se realiza la multiplicación de proteína, grasa, carbohidratos por los factores establecidos según Norma INEN 1334, expresado el Kcal/g.

\section{Determinación de Vitamina C}

Para determinar Vitamina $\mathrm{C}$ se realiza mediante el método de Yodimetría.

\section{Análisis Microbiológicos}

Determinación de mohos y levaduras se realizó mediante bajo la norma (NTE INEN 386).

Análisis de aceptabilidad

Con la finalidad de evaluar la aceptabilidad del producto se utilizó una prueba hedónica, asignando un valor a cada atributo según la categoría reportada en la escala que fue desde "me disgusta mucho" hasta "me gusta mucho"

Se empleó un diseño completamente al azar DCA. El análisis de datos se realizó con ayuda del paquete estadístico Infostat versión 2018. Las diferencias entre tratamientos fueron evaluadas mediante el cálculo del ADEVA y para la separación de medias la 
prueba de Tukey al 5\%. Estadística descriptiva para las pruebas microbiológicas(Velasco A, 2020)

\section{Resultados y discusión.}

\section{Análisis físico-químicos}

Los resultados obtenidos de los análisis físicos químicos de la mermelada hipocalórica de arazá y babaco con diferentes niveles de stevia se reportan en la tabla 1, los mismos que se detallan a continuación.

Tabla 1: Valoración físico químico de la mermelada hipocalórica de arazá y babaco utilizado diferentes niveles de stevia (stevia rebaudiana).

\begin{tabular}{|c|c|c|c|c|c|c|}
\hline \multirow{2}{*}{ Variables } & \multicolumn{4}{|c|}{ Niveles de stevia } & \multirow{2}{*}{ EE. } & \multirow{2}{*}{ Pro. } \\
\hline & $0 \%$ & $2 \%$ & $4 \%$ & $6 \%$ & & \\
\hline $\mathrm{pH}$ & $2,66^{\mathrm{a}}$ & $2,96 a$ & $3,02^{\mathrm{a}}$ & $3,07 \mathrm{a}$ & 0,10 & $0,0617 \mathrm{~ns}$ \\
\hline Acidez \% & $0,18^{\mathrm{a}}$ & $0,18 \mathrm{ab}$ & $0,15 b c$ & $0,14 \mathrm{c}$ & 0,01 & $0,0001 \mathrm{~s}$ \\
\hline $\begin{array}{c}\text { Azucares } \\
\text { Reductores\% }\end{array}$ & $13,92^{\mathrm{a}}$ & $7,58 \mathrm{a}$ & $6,03^{a}$ & $5,01 \mathrm{~b}$ & 0,93 & $0,0001 \mathrm{~s}$ \\
\hline Brix & $68,63^{\mathrm{a}}$ & $15,20 \mathrm{ab}$ & $17,38 b$ & $19,15 \mathrm{c}$ & 0,68 & $0,0001 \mathrm{~s}$ \\
\hline
\end{tabular}

Fuente: Infostat (2019)

Prob. Probabilidad

E.E: Error Estándar

Prob>0,05: no existen diferencias significativas

Medias con una letra común no son significativamente diferentes ( $p>0,05)$

pH: En la tabla 1 se observa los valores de $\mathrm{pH}$ para los niveles de stevia al 0\%,2\%,4\%,6\%, de la mermelada y no presentaron diferencias significativas $(\mathrm{p}<0,05)$, el valor medio es de 2,93 y al compararlo con la (NTE INEN 0419 y Alonso 2012), se indica que el máximo es 3,5, demostrándose que los valores de la investigación están dentro de los parámetros establecidos por dicha norma para la elaboración de mermelada.

Acidez: La acidez de la mermelada en los diferentes niveles de stevia 0\%, 2\%, 4\%, 6\%, presenta diferencias altamente significativas $(\mathrm{p}<0.01)$ para el nivel $6 \%$ se obtiene un valor de 0,14 \% al comparar con la (Resolución 15789 Ministerio de Salud Colombia 1984, citada en Velasco A 2020) quien da un valor mínimo de 0,5\% de acidez y se determina que nuestros resultados cumplen con el valor establecido por dicha norma esto se debe a que las frutas que se utilizaron estaban en estado de madurez comercial además del edulcorante y esto corresponde al contenido de ácidos orgánicos del producto.

Azucares reductores: los azucares reductores presentaron diferencias altamente significativas ( $\mathrm{p}<0,01$ ), obtenido un valor de 5,01\% en el nivel de adición de 6\%, en el estudio realizado por (Flores C, 2012) indica valores de 12,84\%, los cuales no son 
similares a los encontrados, la disminución puede deberse al tipo de edulcorante utilizado pues la cantidad de sacarosa (20-30\% menos) que se utiliza en la elaboración de esta mermelada determina la capacidad de reducción en el oscurecimiento del producto. (García 2013).

${ }^{\circ}$ Brix: Los grados Brix de la mermelada presentaron diferencias altamente significativas ( $\mathrm{p}<0,01$ ), obtenido un valor de $68{ }^{\circ}$ Brix para el nivel $0 \%$ que se encuentra en los parámetros establecidos por la (NTE INEN 0419, 1988) la cual establece $68^{\circ}$ Brix para mermeladas, jaleas y confituras, sin embargo en la norma técnica (NOM-086-SSA1, 1996) nos dice que el contenido de solidos solubles en mermeladas de bajas calorías no debe ser mayor al $25 \%$ y en la investigación se muestran un valor de 19,15 para el nivel del $6 \%$ de stevia la cual está acorde con la norma citada.

Humedad: el contenido de humedad en la mermelada presenta diferencias altamente significativas $(\mathrm{P}<0,01)$ entre los niveles, observándose un valor de $88,15 \%$ para el nivel $6 \%$ de stevia y difiere con los resultados encontrados por (Flores, C, 2012) que obtuvo valores más bajos, esto se debe a que en la mermelada de guayaba se utilizó una formulación tradicional mientras que en la mermelada hipocalórica se disminuyó el porcentaje de azúcar.

Tabla 2: Composición nutricional de la mermelada hipocalórica de Arazá y babaco.

\begin{tabular}{|c|c|c|c|c|c|c|}
\hline \multirow{2}{*}{ Variables } & \multicolumn{4}{|c|}{ Niveles } & \multirow{2}{*}{ EE. } & \multirow{2}{*}{ Pro. } \\
\hline & $0 \%$ & $2 \%$ & $4 \%$ & $6 \%$ & & \\
\hline Humedad & $37,76 a$ & $86,08 b$ & $88,55 \mathrm{c}$ & $88,15 \mathrm{c}$ & 0,38 & 0,0001 \\
\hline Proteína & $2,22 \mathrm{a}$ & $1,33 \mathrm{a}$ & $1,75 b$ & $1,33 c$ & 0,09 & 0,0001 \\
\hline Fibra & $2,37 \mathrm{a}$ & $3,32 b$ & $3,31 \mathrm{~b}$ & $3,29 b$ & 0,04 & 0,0001 \\
\hline Extracto etéreo & $0,13 \mathrm{a}$ & $0,16 b$ & $0,2 \mathrm{bc}$ & $0,19 b$ & 0,01 & 0,0001 \\
\hline Cenizas & $0,86 a$ & $0,38 \mathrm{ab}$ & $0,23 b$ & $0,28 \mathrm{c}$ & 0,03 & 0,0001 \\
\hline ELN & $56,70 \mathrm{a}$ & $7,43 \mathrm{a}$ & $5,97 \mathrm{a}$ & $5,29 b$ & 0,62 & 0,0001 \\
\hline Valor Calórico (Kcal / g) & $236,46 a$ & $18,28 \mathrm{a}$ & $18,75 b$ & $17,81 \mathrm{~b}$ & 1,13 & 0,0001 \\
\hline VIT C $(\mathrm{mg} / \mathrm{kg})$ & $0,03 \mathrm{a}$ & $0,03 \mathrm{a}$ & $0,02 \mathrm{a}$ & $0,03 \mathrm{a}$ & 0,0023 & 0,1678 \\
\hline
\end{tabular}

Fuente: Autores, (2020)

Proteína: El contenido de proteína presento diferencias altamente significativas $(\mathrm{p}<0,01)$, donde el menor porcentaje de proteína es en nivel $6 \%$ con un valor de $1,33 \%$ en comparación con los demás tratamientos, comparando con los resultados de (Castillo, $\mathrm{P}$, 2014 , p) con un valor de 1,86 \% que empleó calabaza correspondiendo al porcentaje de proteína de las frutas utilizadas en la investigación (citado por Velasco, A 2020).

Fibra Cruda: referente al contenido de fibra los tratamientos presentaron diferencias altamente significativas $(\mathrm{p}<0,01)$, donde el nivel $6 \%$ tuvo el valor más alto, la investigación de (Castillo, P, 2014) presentó un porcentaje de $4.82 \%$ en mermelada de 
calabaza observándose valores similares señalando que no se encuentran en los parámetros de (INCAP, 2012), la cual indica un valor de 0,70 en mermeladas toda variedad esto ocurre según (Flores ,C, 2012) porque al eliminar el agua contenida en la fruta durante el proceso de cocción hay una mayor concentración de soluto.

\section{Extracto Etéreo.}

El contenido de extracto etéreo que se reportó en los diferentes niveles presentan diferencias altamente significativas ( $\mathrm{p}<0,01)$, con un valor de $0,19 \%$ en el nivel $6 \%$, en comparación a la investigación (Flores, C, 2012) elaboración y evaluación comparativa de mermelada de guayaba (psidium guajava) deshidratada frente a mermeladas casera e industrial las cuales reportan valores similares resultados correspondientes principalmente a vitaminas liposolubles y carotenoides (Duran S. 2012, Citado en Velasco A., 2020); no obstante en el Ecuador no existen parámetros de grasa en la normativa para su comparación.

Cenizas: En el contenido de cenizas los resultados obtenidos tuvieron diferencias altamente significativas $(\mathrm{p}<0,01)$, teniendo un valor de $0,28 \%$ para el nivel $6 \%$, el porcentaje de azúcar blanca pudo influir en los resultados, elevando la concentración de minerales sin embargo relacionando los datos de la investigación con (Gamarra , J y Rosales, A, 2016) quienes realizaron mermelada dietética de piña y naranja obtuvieron valores de $0,41 \%$ la cual difiere con los resultados obtenidos debido al tipo de edulcorante y el porcentaje de adición de azúcar. (García J, 2016 citada en Velasco A 2020)

\section{Extracto Libre Nitrogenado.}

El contenido de ELN como presento diferencias altamente significativas ( $p<0,01)$, el nivel $0 \%$ con un valor de $57,70 \%$ tiene un mayor contenido de carbohidratos en comparación con los demás tratamientos, de acuerdo a (INCAP, 2012), señala un valor de $66,30 \%$

\section{Contenido calórico.}

El contenido calórico presentó diferencias altamente significativas $(\mathrm{p}<0,01)$, entre los tratamiento, observándose un aporte energético de $(236,46) \mathrm{kcal} / \mathrm{g}$ en el nivel $0 \%$, la cual cumple los parámetros de la norma técnica (INCAP, 2012), en el que se establece un valor de $246 \mathrm{kcal} / \mathrm{g}$ para mermeladas de toda variedad y al comparar con la investigación de (Espín , X, 2012 ) quien utilizó zanahoria amarilla (Daucus carota) con manzana y diferentes concentraciones de pectina obtuvo un valor de $307 \mathrm{kcal} / \mathrm{g}$ esto se debe al porcentaje de azúcar utilizado; además del estado de madurez de la fruta que se empleó, sin embargo en los demás tratamientos el porcentaje de calorías se redujo notablemente en comparación con el nivel 0\% y de acuerdo a la norma (NOM-086-SSA1, 1996, DOROSZ) señala un valor de $40 \mathrm{kcal} / \mathrm{g}$ como máximo, es así que el nivel $6 \%$ presenta un valor de 17,81 Kcal /g cumpliendo con la norma. 


\section{Análisis microbiológicos.}

Al evaluar el análisis microbiológico de la mermelada niveles de stevia se pudo evidenciar ausencia total de mohos y levaduras. El método de ensayo que se utilizó para determinar mohos y levaduras fue bajo la norma (NTE INEN 386: 1985) y según las referencias de (Esmeralda J 2018).

\section{Pruebas de aceptabilidad.}

Se realizó una prueba de aceptabilidad escala hedónica en las categorías de me agrada mucho, me disgusta, no me gusta ni me disgusta, me gusto, me disgusta.

Color: Para determinar la aceptabilidad del color de la mermelada se realizó una prueba a los catadores para este parámetro, como se puede observar en el grafico 1, se tuvo una aceptabilidad en la categoría de me gusta mucho para el nivel $0 \%$ con el 50\% la cual nos dice que el color de la mermelada es aceptable sin embargo para la categoría de no me gusta ni me disgusta se obtuvo un valor de 50\% para el nivel $4 \%$, de acuerdo a (Otiniano „J 2017) posiblemente se debería a una cocción prolongada y al estado de madurez de la fruta. (Velasco, A 2020)

Gráfico 1: Análisis del parámetro de Color

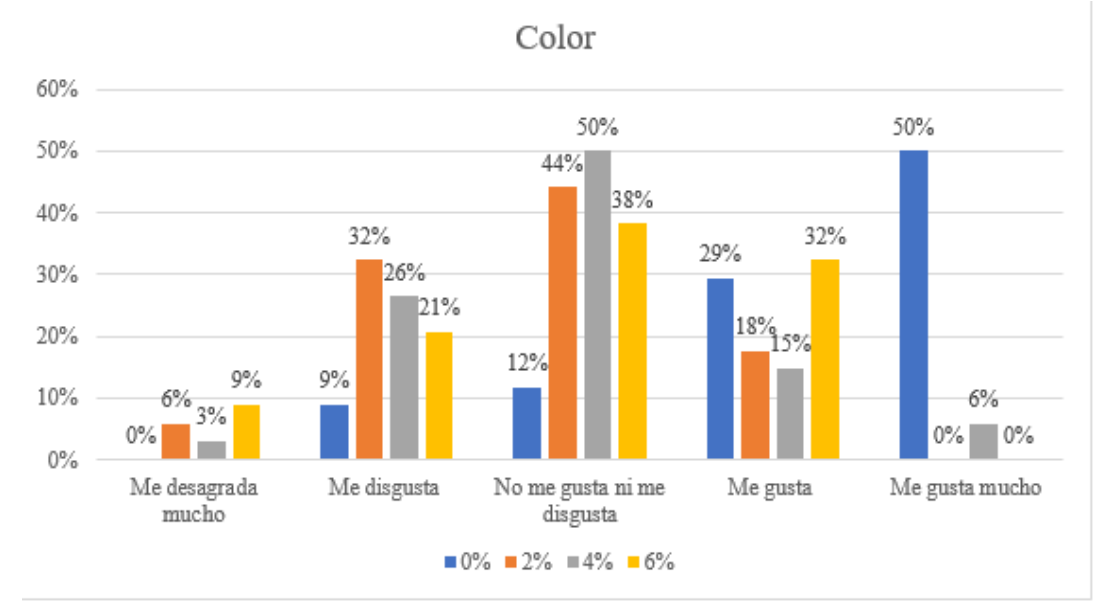

Fuente: Autores, (2020).

Olor: De acuerdo del parámetro de olor se determinó una mayor aceptabilidad para el nivel $6 \%$ con un $41 \%$ como se puede observar en el grafico 2 en la categoría de me gusta y en la de no me gusta ni me disgusta, seguidos por el nivel $4 \%, 0 \%$ con el $38 \%$, y el puntaje más bajo fue en el nivel $0 \%$ con $35 \%$ en la misma categoría, sin embargo en la categoría de me gusta mucho se observa un valor de $35 \%$ lo que se entiende que no influye la formulación de la mermelada. (Velasco, A 2020) 
Gráfico 2: Análisis del parámetro olor.

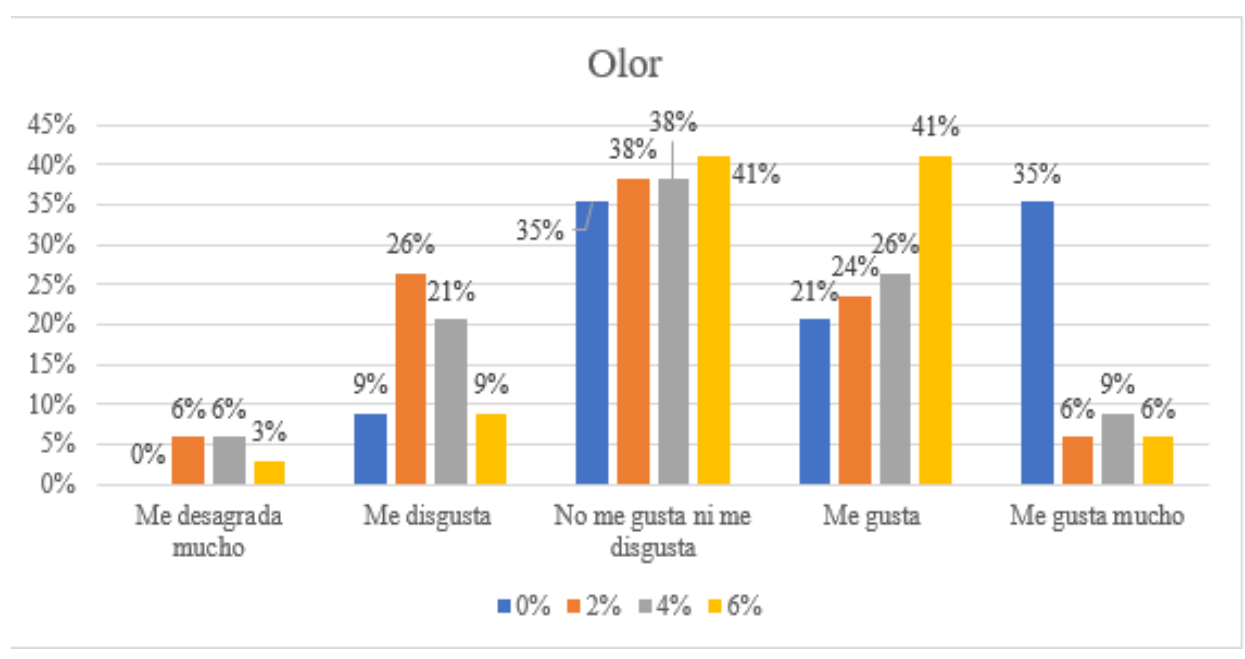

Fuente: Autores, (2020)

\section{Sabor.}

El puntaje en la categoría de me gusta fue 38\% para el nivel $6 \%$ y existe una coincidencia de valores con el nivel $0 \%$ con igual puntaje en la categoría de me gusta mucho, lo cual indica que es aceptable y no existen cambio ni factores externos que influyan en su sabor de acuerdo a las respuestas de los catadores para este nivel, además de diferir en el nivel $2 \%$ la cual tiene un valor de $41 \%$ en la categoría de me disgusta lo que se atribuye al porcentaje de stevia utilizado en la elaboración de la mermelada. (Velasco, A 2020)

\section{Conclusiones:}

- Se pudo elaborar mermeladas de arazá y babaco utilizando diferentes niveles de stevia (stevia rebaudiana) 2, 4 y $6 \%$, en donde el nivel $6 \%$ presenta el menor valor calórico $17 \mathrm{kcal} / \mathrm{g}$ (20 Kcal/g para etiquetado) en relación al tratamiento testigo para el que se calculó un valor de 236,46 kcal/ g (240 kcal/g para etiquetado).

- Las mejores características nutricionales, sensoriales y microbiológicas se atribuyen al nivel 6\% de adición de stevia debido a su bajo aporte calórico.

- Se evaluó la composición nutricional en todas las mermeladas, donde el nivel 6\% presento valores de $88,15 \%$ de humedad, 1,33\% proteína, 3,29\%, 3,29\% Fibra, $0,19 \%$ extracto etéreo, $0,28 \%$ minerales totales y $5,29 \%$ extracto libre nitrogenado, siendo el nivel con mayor (olor color y sabor), microbiológicamente los tres niveles no presentaron crecimiento de microorganismos. 


\section{Referencias bibliográficas:}

Aoac. (2005). Official Methods of Analysis of AOAC INTERMATIONAL. 18th ed. Association of Official of Analytical Chemist. Washington, DC

Alonso, Iván. Determinación de pH y acidez Titulable total [blog]. Peru: David Ramirez, 12 de 11 del 2012, 2012.[Consulta: 19 de Junio de 2019]. Disponible en: https://www.academia.edu/22698699/._determinaci\%c3\%93n_de_ph_y_acidez_ titulable_en_los_alimentos.

Castillo Gavidia, Patricia Valeria. "Efecto del uso de dos edulcorantes naturales ( Stevia y Panela ) sobre el poder calorico de una mermelada de calabaza" . [En línea] (Tesis pregrado). Universidad Tecnológica Equinoccial Sede Santo Domingo, Carrera De Ingeniería Agroindustrial Y Sistemas De Gestión, Santo DomingoEcuador, 2014. p. 38. [Consultado: 15 de 12 de 2019.]. Disponible en:http://192.188.51.77/bitstream/123456789/19139/1/7243_1.pdf.

Cuèllar, Eliana \& Jimenez, Claudia. Caracterización Física Y Química Del Fruto De Arazá (Eugenia stipitata Mc Vaugh). Ingenieria \& Amazonas [En línea], 2013, Bogota- Colombia, 6(2), p. 117. [Consultado: 19 de 10 de 2019.]. ISSN 16927389. Disponible en: http://www.udla.edu.co/revistas/index.php/ingenierias-yamazonia/article/view/329.

Dorosz. Tabla de Calorias . [Libro electronico]. decimo tercera edicion. BarcelonaEspaña : HISPANO EUROPEA , 1998. [Consultado: 17 de 11 de 2019.] Disponible en: https://books.google.es/books?id=RW0zo5c_Y48C\&\%20printsec.

Duran Aguero, Samuel, Rodriguez N, Cordon, Maria del Pilar. Estevia (stevia rebaudiana), edulcorante natural y no calórico. Revista Chilena de nutricion [En línea], 2012, Santiago-Santiago de Chile 39(4). p. 204. [Consultado: 05 de 11 de 2019.] ISSN 0717-7518. Disponible en: https://scielo.conicyt.cl/pdf/rchnut/v39n4/art15.pdf.

Esmeralda Gamboa, Jenniffer Gabriel \& NAZARENO ARROYO, Jennifer Isabel. Estudio bromatológico de la especie ecuatoriana arazá (Eugenia stipitata) de diferente origen geografico.[En línea] (Tesis pregrado). Universidad De Guayaquil, Facultad De Ciencias Químicas, Escuela De Química Y Farmacia, Guayaquil- Ecuador. 2018. p.11. [Consultado: 13 de 11 de 2019.]. Disponible en:http://repositorio.ug.edu.ec/handle/redug/33634.

Espin Tapia , Mayra Ximena ."Uso de la zanahoria amarilla (Daucus carota) mediante una mezcla con manzana a diferentes concentraciones de pectina para elaborar una mermelada". [En línea] (Tesis pregrado). Universidad Tecnica de Ambato, Facultad de Ciencias e Ingenieria en Alimentos Carrera de Ingenieria en Alimentos, Ambato- Ecuador. 2012. p. 52. [Consultado: 18 de 11 de 2019.]. Disponible en:http://repositorio.uta.edu.ec/bitstream/123456789/3131/1/S.AL486.pdf. 
Flores Iturralde, Carla Jacqueline. "Elaboracion y evaluacion nutricional comparativa de mermelada de guayaba((Psidium guajava) deshidratada frente a mermeladas caseras e industrial". [En línea] (Tesis pregrado). Escuela Superior Politécnica De Chimborazo, Facultad De Ciencias, Escuela De Bioquímica Y Farmacia, Riobamba- Ecuador. 2012, p. 5-75. [Consultado: 19 de 11 de 2019.]. Disponible en: http://dspace.espoch.edu.ec/bitstream/123456789/2470/1/56T00354.pdf.

Gamarra Condor, Jose Luis, \& Rosales Mateo , Ana Rosa . "Caracterizacion de la mermelada dietetica de piña (Anana Comusos) y naranja (Citrus sinensis) edulcorado parcialemente con stevia (Stevia rebaudiana)". [En línea](Tesis pregrado). Universidad Nacional Del Centro Del Perú, Facultad De Ingeniería Y Ciencias Humanas, Escuela Académico Profesional De Ingeniería Agroindustrial, Junin- Peru. 2016. p. 122. [Consultado: 18 de 11 de 2019.]. Disponible en:http://repositorio.uncp.edu.pe/handle/UNCP/4741.

Garcia Almeida, Jose Manuel, Casado Fdez, Gracia M \& Garcia Aleman, Jose . Una vision global y actual de los edulcorantes. Aspectos de regulación. Nutricion Hospitalaria [En línea] 2013, (España) 28(4), pp. 17-22. [Consultado: 20 de 11 de 2019.]. Disponible enhttp://scielo.isciii.es/pdf/nh/v28s4/03articulo03.pdf.

Instituto De Nutrición De Centro América E Panamá (INCAP). Tabla de composicion de alimentos de centroamérica. Cuidad de Guatemala- Guatemala. 2012,p. 54 [Consultado: 15 de 12 de 2019.]. Disponible en: http://www.incap.int/mesocaribefoods/dmdocuments/TablaCAlimentos.pdf.

INEN 0419. Instituto Ecuatoriano de Normalización. Norma Técnica Ecuatoriana. Conservas Vegetales, Mermeladas De Frutas. Requisitos. Quito- Ecuador. 1988, p. 1-4. [Consultado: 18 de 11 de 2019.]. Disponible en: https://ia801601.us.archive.org/2/items/ec.nte.0419.1988/ec.nte.0419.1988.pdf.

INEN 2825. Instituto Ecuatoriano de Normalización. Norma Técnica Ecuatoriana. Norma para las confituras, jaleas y mermeladas(codex stan 296-2009, mod). QuitoEcuador. 2013, p.1 [Consultado: 18 de 11 de 2019.]. Disponible en:https://181.112.149.204/buzon/normas/nte-inen-2825.pdf.

INEN 386. Instituto Ecuatoriano De Normalización. Norma Técnica INEN 386. Conservas vegetales ensayos microbiologicos mohos. Quito- Ecuador. 2013, p.12 [Consultado: 5 de 11 de 2019.]. Disponible en: https://181.112.149.204/buzon/normas/386.pdf

NOM-086-SSA1. Norma Oficial Mexicana. Bienes y servicios. Alimentos y bebidas no alcohólicas con modificaciones en su composición. Especificaciones nutrimentales. Cuidad de mexico-Mexico. 1996, p.7 [Consultado: 04 de 11 de 2019.]. Disponible en: http://www.economianoms.gob.mx/normas/noms/1996/086-ssa1.pdf.

Otiniano Verde Julissa Selene, "Elaboracion y evaluacion reologica de mermelada de naranjilla". [En línea] ( Tesis pregrado). Universidad Nacional Agraria De La Selva, Facultad De Ingeniería En Industrias Alimentarias, Departamento Académico De Ciencia, Tecnología E Ingeniería De Alimentos, Tingo María - 
Perú. 2017, p. 13-19. [Consultado: 18 de 11 de 2019.]. Disponible en: http://repositorio.unas.edu.pe/handle/UNAS/1257.

Resolucion 15789. Republica De Colombia Ministerio De Salud. Características Organolépticas físico- químicas y microbiológicas de las mermeladas y jaleas. Bogota- Colombia. 1984, p. 1 [Consultdado: 5 de 11 de 2019.]. Disponible en: https://rosdary.files.wordpress.com/2008/03/resolucion-15789-de-1984.pdf.

Rojas Parra Sol Ángela, et al. Stevia "El dulce sabor de tu vida ". [blog]. Bogota Colombia 2007, p. 2-20 [Consultado: 25 de 11 de 2019.]. Disponible en: http://www.agrolalibertad.gob.pe/sites/default/files/manual\%20stevia.pdf

Velasco Duche, Adela. "Elaboracion y evaluacion reologica de mermelada de naranjilla". [Documento físico] (Tesis pregrado). Escuela Superior Politécnica de Chimborazo, Facultad De Ciencias Pecuarias. Carrera de Industrias Pecuaria Ecuador. 2020, p. 35-60. [Consultado: 18 de 02 de 2020.]. 
PARA CITAR EL ARTÍCULO INDEXADO.

López Sampedro, S. E., Velasco Duche, A. M., Baño Ayala, D. J., \& Paredes Peralta, A. V. (2020). Mermelada hipocalórica de arazá y babaco. ConcienciaDigital, 3(3), 151-163. https://doi.org/10.33262/concienciadigital.v3i3.1287

\section{\Ciencia}

El artículo que se publica es de exclusiva responsabilidad de los autores y no necesariamente reflejan el pensamiento de la Revista Conciencia Digital.

El artículo queda en propiedad de la revista y, por tanto, su publicación parcial y/o total en otro medio tiene que ser autorizado por el director de la Revista Conciencia Digital.
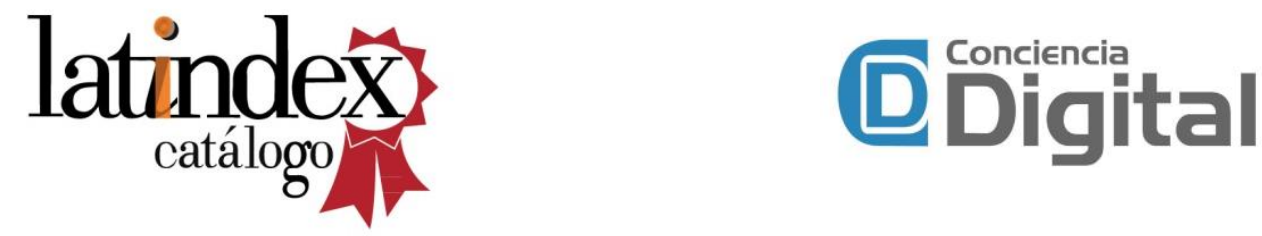